\title{
Lebensqualität bei Varicosis
}

Lumley E et al. Experiences of living with varicose veins: a systematic review of qualitative research. J Clin Nursing 2019; 28: 1085-1099. doi:10.1111/ jocn. 14720

5-15\% der Männer und bis zu jede 3. Frau leiden unter Krampfadern (VV). Die Entscheidung für oder gegen eine Intervention hängt stark vom subjektiven Leidensdruck ab. Fachgesellschaften empfehlen deshalb, die individuellen Belange der Patienten zu berücksichtigen und mit PROMs (Patient Related Quality of Life) zu erfassen. PROMs enthalten Batterien strukturierter Fragen zur Gesundheit und Lebensqualität aus Sicht der Erkrankten. In Großbritannien kommen solche Tests bei VV präoperativ zum Einsatz, wobei die Inanspruchnahme unter der bei anderen Interventionen blieb. Die Arbeitsgruppe um Lumley stellte die Frage, ob die für die VV verfügbaren PROMs tatsächlich die Symptome und Belange der Patienten reflektieren. In verschiedenen elektronischen Datenbanken fanden sich 3 qualitative Studien, die die Einschlusskriterien erfüllten, in einer thematischen Synthese zusammengeführt und verglichen wurden. Die Explorationen erfolgten $u$. a. nach einem phänomenologischen Ansatz, mit semistrukturierten Interviews und Framework-Analyse. Die Da- tenextraktion wurde mit der CASP-Checkliste durchgeführt (Critical Appraisal Skills Programme qualitative research checklist).

5 Themen stellten sich als Schwerpunkte heraus: körperliche Auswirkungen der VV, psychische Folgen, der Einfluss auf das soziale Leben, die Anpassung an die Krankheit und Gründe für einen Behandlungswunsch. Die physischen Symptome waren vielfältig, wobei Schmerzen, Schweregefühl und Juckreiz am häufigsten genannt wurden. Die Beschwerden wirkten sich oft auf andere Aspekte aus und führten z. B. zu Schlafmangel und Erschöpfung. Die Patienten setzten unterschiedliche Strategien ein (Hochlegen der Beine, Cremes und Kompressionsstrümpfe). Die 2 Studien, in denen über Kompressionsstrumpfhosen berichtet wurde, kamen zu verschiedenen Ergebnissen. Der abschwellenden Wirkung und Erleichterung bei der Arbeit standen eine weitgehende Unzufriedenheit gegenüber. Die Studien ergaben, dass die Patienten durch die VV in ihrer Funktionalität beeinträch- tigt waren; dies galt bei der Arbeit und für Freizeitaktivitäten („not being able to live fully“). Psychologisch resultieren Ängste vor möglichen Komplikationen wie tiefen Beinvenenthrombosen und Ulcera. Die Sorgen um das Aussehen führten zu Verlegenheit, Ekel und einem reduzierten Selbstwertgefühl. Dies galt für Männer und Frauen und mündete $u$. $U$. in veränderten Freizeitaktivitäten, bedeckender Kleidung und sozialem Rückzug. Hauptmotivation für den Wunsch nach einer Therapie waren die Hoffnung auf eine Symptomerleichterung und den Schutz vor Komplikationen. Die Erwartungen an den Nutzen waren dabei oft unrealistisch.

Die Zusammenschau und der Vergleich zeigten, dass die bislang validierten PROMs den subjektiven Einfluss einer VV auf die Lebensqualität nicht in Gänze abbildeten. Im Gesundheitswesen Tätige sollten die ganze Bandbreite der subjektiven Auswirkungen einer VV kennen, so Lumley.

Dr. med. Susanne Krome, Melle 